\title{
How Well Do Ontario Library Web Sites Meet New Accessibility Requirements?
}

\author{
Joanne Oud \\ Instructional Technology Librarian \\ Wilfrid Laurier University Library \\ joud@wlu.ca
}

\begin{abstract}
New changes to Ontario law will require library web sites to comply with the Web Content Accessibility Guidelines, version 2.0 (WCAG 2.0). This study evaluates 64 Ontario university, college, and public library web sites to see how well they comply with WCAG 2.0 guidelines at present. An average of 14.75 accessibility problems were found per web page. The most common problems included invalid html, poor color contrast, incorrect form controls and labels, missing alt text, bad link text, improper use of headings, using html to format pages, using absolute units of measure, and issues with tables and embedded objects.
\end{abstract}

\section{Keywords}

accessibility; web; disabilities; laws; guidelines; academic libraries; public libraries; web site design

The Accessibility for Ontarians with Disabilities Act (AODA) has been augmented with new standards which require improved accessibility for people with disabilities. These standards set out specific guidelines and requirements for accessibility in a variety of areas. The Customer Service Standard took effect Jan 1, 2012 for all organizations with more than one employee, and mandates accessible service for businesses, schools and libraries in Ontario. Standards for Transportation, Human Resources, Built Environment, and Information and Communication have been merged into a single Integrated Standard which was passed into law in June 2011. The Information and Communication section of the Integrated Standard sets out requirements for providing information in accessible formats and will have a significant impact on libraries, including their collections, their method of communicating with patrons, and their web sites. By 2021, all Ontario web sites will need to comply with established international standards for web accessibility.

The new standards are based on the principles of ensuring independence and dignity. As of 2011, one in seven people in Ontario had a disability (Ontario Ministry of Community and Social Services, "Understanding Accessibility"), and that number is growing. By definition, disabilities include physical, visual and hearing impairments, psychological impairments such as depression or anxiety disorders, and learning or cognitive impairments. The new standards attempt to ensure that people with these disabilities can do what everyone else does, without requiring assistance, at the same time that everyone else can do it. The standards are based 
on contemporary ideas about disability which recognize that the environment creates disability by putting up barriers that make it impossible for people to do things independently (Schmetzke 454). This perspective focuses on problems in the environment rather than on perceived problems with people who have disabilities. A web site which doesn't allow a person with vision impairments to access content puts that person into a disabling situation created by the web site. The new standards attempt to create a more level playing field for people with disabilities by removing barriers in the environment that prevent them from full access to society (Ontario Ministry of Community and Social Services, "Understanding Accessibility"). In libraries, web sites play a critical role in providing access to information. If those web sites are not accessible, libraries "bear the responsibility for putting in place conditions that are disabling for some of our users" (Schmetzke 455), and for creating a digital divide where some people do not have access to resources and services.

The new AODA Information and Communication Standard is meant to prevent this situation. The Standard covers all types of information and communication, including library web sites. It specifies that all existing web sites must comply with the Web Content Accessibility Guidelines version 2.0 (WCAG 2.0) by 2021. WCAG 2.0 has three levels or priorities, and the Standard specifies compliance with the first two levels: $A$, which is the most basic, and $A A$, which is more advanced. Though all sites must comply by 2021, any new sites created after January 1, 2014 or any existing sites substantially revised after that date must comply with WCAG 2.0 level A guidelines. In addition, after January 1, 2012, any new content created on existing web sites must meet WCAG 2.0 level A guidelines. This applies to any web content or pages libraries create or have control over "through a contractual relationship that allows for modification of the product" (Ontario Ministry of Community and Social Services "Integrated Standard"). This includes any web pages generated through content management systems, or content libraries get through vendors but can modify: for example, pages generated from purchased software such as LibGuides or Serials Solutions.

WCAG 2.0, the guidelines set out in the Standard, are the most up to date, comprehensive set of web accessibility guidelines available, are internationally recognized, and tend to be standard advice in works on web accessibility. The guidelines consist of two major parts: a document created in 2008 outlining general principles for web accessibility (World Wide Web Consortium (W3C), "Web Content Accessibility Guidelines (WCAG) 2.0."), and a 2010 document setting out specific techniques for implementing these principles, along with criteria for evaluating whether they have been successfully met or not (World Wide Web Consortium (W3C), "Techniques and Failures for Web Content Accessibility Guidelines (WCAG) 2.0.").

Fortunately for Ontario web developers, the Information and Communication Standard requires compliance with web accessibility guidelines that are broadly recognized. WCAG 2.0 is still relatively new, however, and since not all jurisdictions require compliance to the same guidelines, existing advice in the library and web development literature is often not applicable. In fact, the library literature does not contain much advice on the accessibility of library-created web sites or on conforming to WCAG 2.0 standards. The largest number of studies on library web accessibility deal with library resources. A number focus on the accessibility of 
databases licensed from vendors (Stewart, Narendra and Schmetzke; Tatomir and Durrance; Power and LeBeau; Byerley, Chambers and Thohira; Dermody and Majekodunmi), and one on the accessibility of the online catalogue interfaces provided by major vendors (Shaughnessy). A few studies focus on the accessibility of non-library web sites. Jaeger studies the accessibility of U.S. government web sites, and Brophy \& Craven summarize existing large-scale studies of web site accessibility in the U.K. Only a few studies focus specifically on the accessibility of web sites created by libraries. One study reported on automated testing of the library web sites from mid-sized universities in the U.S. and found that $58 \%$ were inaccessible (Spindler 151). Another study looked at the accessibility of 29 U.S. and Canadian university library web sites and found an average of 3.6 accessibility errors per page, with Canadian libraries having slightly fewer than U.S. libraries (Comeaux \& Schmetzke 465). This error rate was somewhat better than a similar study done by the same authors five years previously, but the researchers were still forced to conclude that library web sites "tend to disregard established accessibility guidelines"(Comeaux \& Schmetzke 475). A third study evaluated the accessibility of academic library web sites in Kentucky and found that only one library (3\%) was compliant with U.S. Section 508 accessibility guidelines (Providenti and Zai 488). None of these studies evaluated for accessibility using WCAG 2.0 guidelines, which were finalized in late 2008.

Some work sheds light on the barriers to creating accessible web sites. Brophy and Craven point out that web site creators tend to have some awareness of accessibility, but that studies have shown that they lack knowledge of specific accessibility issues, guidelines, and techniques for implementing them (968). Jaeger surveyed U.S. government web site creators and found that they generally felt that their sites were more accessible than they were (185).

The studies that do discuss accessibility problems with library web sites tend not to identify specific errors in any detail. Spindler and Comeaux and Schmetzke focus on the number of errors per page but not on which errors were found. Providenti and Zai do point out a few common errors, including invalid html code and missing alt text (484), but their evaluation is done based on U.S. Section 508 guidelines rather than WCAG 2.0. Some articles give tips on creating accessible web sites (Kirkpatrick; Murley; Hein and Seyfarth), but these are not based on WCAG 2.0 guidelines and do not contain data on which problems are most likely to be encountered on library web sites.

These existing studies suggest that there are likely to be a number of accessibility problems on university library web sites. Unfortunately, there is a lack of information on the Ontario library situation, on public and community college library web site accessibility, on the conformance of library web sites in general to WCAG 2.0 guidelines, and on the types of accessibility problems most likely to be found on library web sites. Without this information, it will be difficult for Ontario libraries to determine how to start to comply with the new accessibility legislation. Therefore, the goals of this research project are to determine how compliant Ontario public, community college, and university libraries are with WCAG 2.0 guidelines at present and to identify the major compliance errors that will need to be corrected. 


\section{Evaluation Methods}

This study evaluated university, college, and major public library web sites in Ontario to determine compliance with WCAG 2.0 guidelines, A and AA priority. All members of the Ontario Council of University Libraries (OCUL) were included. The community colleges were taken from the list on the Ontario Colleges application site. Three were not evaluated because their web sites were too small, consisting of only one web page. Three share a web site with a university, so were not analyzed separately. The remaining college sites were included in the analysis. In addition, the study analyzed the web sites of the public library system of any Ontario municipality with a population of over 100,000 as determined by the most recent available Canadian Census data from 2006. A total of 64 library web sites were analyzed: 20 university, 21 college, and 23 public.

An automated web accessibility checking tool was used to analyze the sites during the summer of 2011. Since the study results depend on the accuracy of the tool used, a number of tools that check against WCAG 2.0 standards were tested, including Qompliance, SortSite, Total Validator, TAW3, Wave, Functional Accessibility Evaluator (FAE), and AChecker. Total Validator (professional version) was selected because it had the lowest false positive error reporting and because it performed the most comprehensive analysis of WCAG 2.0 errors.

There are inherent limitations to using automated accessibility checkers, which have been mentioned by other researchers (Jaeger 181; Spindler 151; Comeaux and Schmetzke 474). The most obvious limitation is that such checkers can only evaluate guidelines that can be seen clearly in the web page's html code. A significant number of WCAG 2.0 guidelines, such as consistent use of terminology, simplicity of interface, or clear language, can't be evaluated in this way. This means that any account of a site's accessibility done using an automated accessibility checker will be incomplete. Using an automated checker does identify a substantial number of problems. Any detailed analysis of an individual site will also need to include expert and user testing, however, since a page which shows no errors when evaluated using an automated checker is not necessarily accessible or compliant with WCAG 2.0 .

Total Validator was used to assess the home page and 29 other pages from each site, randomly selected by Total Validator, for a total of 30 pages per site or 1,860 pages overall. If a site had less than 30 pages, all pages were checked. Total Validator was set to check all pages for markup errors in the page's html/xhtml and css code and WCAG 2.0 accessibility errors. Pages from library catalogues and vendor databases were not checked since the library does not have control over these pages. Pages requiring a login were not checked for practical, logistical reasons. Pages generated from externally purchased or licensed sources, such as LibGuides or Serials Solutions, were included in the analysis since these are normally presented as part of the library's web site in the same way as other locallyproduced pages. All pages from vendors that allow for local modification need to be accessible under the new regulations. Since libraries typically do have some control over the format and design of these pages, they need to conform to WCAG 2.0 guidelines under the Information and Communication section of the Integrated Standard. 
In addition, each page checked by Total Validator was separately analyzed by a semi-automated tool called WCAG Contrast Checker which checks pages for color contrast by analyzing the colors specified in the page markup. WCAG Contrast Checker is available as a Firefox add-on and uses the ratios for minimum color contrast as specified in WCAG 2.0, which vary by size and weight of text.

Errors reported in the results include any error in the html/xhtml or css code or any WCAG 2.0 priority A or AA error as presented by Total Validator and any color contrast error as identified by WCAG Contrast Checker. The number of instances of each error was disregarded in the analysis, although it was common for the same error to be repeated more than once on a page. This means that, even if an error was present multiple times on a page, it was counted as one error.

\section{Results \& Discussion Compliance and Error Rates}

Each of the 1,860 pages checked had errors, which means that none of the web sites are in compliance with WCAG 2.0 guidelines. Table 1 summarizes the number of errors and breaks errors down by library type. On average, across all library types, the analysis found 14.75 accessibility errors per page. These errors can be broken down into an average of 5.79 markup errors (html/xhml or css), 3.28 color contrast errors, and 5.68 other WCAG 2.0 errors per page.

Table 1. Average number of accessibility errors per page

\begin{tabular}{|l|l|l|l|l|}
\hline Library Type & Markup errors & Contrast errors & $\begin{array}{l}\text { Other WCAG } \\
2.0 \text { errors }\end{array}$ & Total errors \\
\hline All libraries & 5.79 & 3.28 & 5.68 & 14.75 \\
\hline College libraries & 6.52 & 3.50 & 5.97 & 15.99 \\
\hline Public libraries & 5.92 & 3.31 & $5.15^{*}$ & 14.38 \\
\hline $\begin{array}{l}\text { University } \\
\text { libraries }\end{array}$ & $4.96^{*}$ & 3.03 & 6.00 & 13.99 \\
\hline
\end{tabular}

${ }^{\star}=$ significant difference $(\operatorname{sig}=.00)$

This error rate is considerably higher than that reported by Comeaux and Schmetzke, which was an average of 3.6 errors per page (465). However, their analysis did not check for markup or contrast. When markup and contrast errors are excluded, the average error rate in this study was 5.68 compared with Comeaux \& Schmetzke's 3.6. Other studies have found that web sites have become progressively more complex, and that site accessibility has tended to decrease as complexity increases (Hackett, Parmanto and Xiaoming 415). This trend towards increased complexity could partly explain the higher rate of errors found in the present study. However, the Comeaux and Schmetzke study evaluated pages against WCAG 1.0 rather than the later WCAG 2.0 standard and used Bobby, an automated checking tool which is now defunct. These factors could also account for the difference in error rate. 
Analysis was done to see if there were differences in error results by page type or type of library. Since the dataset did not have a normal distribution, non-parametric tests were used. For page type, error rates on home pages, which form the main entry point to a site, were compared with error rates on other pages. The comparison found significant differences (Mann-Whitney test, sig =.000). Home pages had a significantly higher number of accessibility errors than other pages, although there were no significant differences in the rates of markup or contrast errors. The larger number of accessibility errors on home pages is a concern since these pages are the primary entry point to library resources and services for users.

Error rates of public, college and university library web sites were compared by library type. Some significant differences were found (Kruschal-Wallis test, sig = .00). Public libraries had a significantly lower number of accessibility errors (excluding markup and contrast errors) than college or university libraries (mean rank: university 974.15, college 1006.80, public 827.74). University libraries had a significantly lower number of page markup errors than college or public libraries (mean rank: university 741.31, college 1090.97, public 962.06). More research would be needed to identify possible factors leading to these differences by library type.

Interpreting these numbers is challenging. The number of errors gives some indicator of the level of accessibility of a page but doesn't show the impact of the errors found or their severity. This means that a page with 10 errors is not necessarily less accessible than a page with 3 , since certain errors render a page completely unusable. One example is automatic page refresh: people using screen reading software take longer than others to read the page, and if the page refreshes as they are reading, they are taken back to the beginning of the page and need to start over each time. Other errors are less severe in their impact, such as using the same link text for different links. Their presence may make the page inconvenient or challenging to use, but people can still largely access the content. Many kinds of accessibility problems, as mentioned previously, can't be checked by automated tools and therefore are not accounted for in the error rates reported in this study. For this reason, absolute measures of web page accessibility are difficult to evaluate.

Therefore, the number of errors on a page is not a reliable indicator of the severity of the accessibility problems in a site, and an error rate of 0 doesn't mean that the page is necessarily accessible. Since Ontario laws will require WCAG 2.0 conformance, however, the relative impact of accessibility errors is not relevant for the purposes of this study. According to Ontario laws, libraries need to comply with all WCAG 2.0 priority $A$ and $A A$ requirements, and WCAG 2.0 compliance requires the absence of any WCAG 2.0 A and AA errors. None of the web sites evaluated came close to meeting these requirements.

\section{Types of Errors}

The most common WCAG 2.0 compliance errors found in web sites in this study are summarized in Table 2. By far the most common error was incorrect html/xhtml or css markup in the page code, which prevents screen reading software from correctly reading the page. Poor contrast between text and background, which makes text hard to read for people with vision impairments, was the second most common error. Using absolute units of measure, such as pixels, to specify sizes on a page was another common error; this prevents people with low vision from enlarging the 
elements on a page to make them readable. Other common errors include not providing alt text for images, which prevents people with visual impairments from interpreting the content of the image, and having multiple links with the same link text on a page. Since people who use screen reading software often navigate using links out of context, repeated link text makes links indistinguishable.

Failure to use html code to create an easily understandable page structure resulted in a number of the other common errors. For example, although basic web design and accessibility guidelines specify separating page formatting from page structure by using css to do formatting, many pages used html to do formatting instead of css. Many others used headings incorrectly for page formatting rather than to indicate the hierarchy of information on the page.

Forms and tables accounted for many of the remaining errors. These include missing or incorrect labels on form fields, which make it difficult for screen reading software to identify the fields, and not including a submit button for forms or form fields such as search boxes. Many tables were missing descriptive text that makes it possible for screen reading software to interpret them.

During analysis, it became evident that each site tended to have similar errors on each of that site's pages. Since all sites appeared to use a single, fairly consistent site template, this finding makes sense. It appears that the majority of errors could be eliminated by fixing the template for each site.

A more detailed explanation of the most common errors follows, with suggestions taken from WCAG 2.0 on how to fix each error and references to the corresponding WCAG 2.0 success criteria (World Wide Web Consortia (W3C), "Techniques and Failures for Web Content Accessibility Guidelines 2.0") to facilitate lookup for further explanations.

Table 2. Most common errors

\begin{tabular}{|l|l|l|}
\hline Error & $\begin{array}{l}\text { \# of Pages with } \\
\text { Errors }(\mathrm{n}=1860)\end{array}$ & $\begin{array}{l}\text { \% of Total Pages } \\
\text { with Errors }\end{array}$ \\
\hline $\begin{array}{l}\text { Invalid page code } \\
\text { (html/xhtml, css) }\end{array}$ & 1857 & 99.8 \\
\hline Poor color contrast & 1681 & 90.4 \\
\hline $\begin{array}{l}\text { Incorrect form control } \\
\text { labelling }\end{array}$ & 1263 & 67.9 \\
\hline Links with same text & 1118 & 60.1 \\
\hline $\begin{array}{l}\text { No alt attributes for } \\
\text { non-text content }\end{array}$ & 1000 & 53.8 \\
\hline $\begin{array}{l}\text { Improper use of } \\
\text { headings }\end{array}$ & 951 & 51.1 \\
\hline $\begin{array}{l}\text { Use of html instead } \\
\text { of css for formatting }\end{array}$ & 894 & 48.1 \\
\hline
\end{tabular}




\begin{tabular}{|l|l|l|}
\hline $\begin{array}{l}\text { Use of absolute units } \\
\text { of measure }\end{array}$ & 738 & 39.7 \\
\hline $\begin{array}{l}\text { No submit button for } \\
\text { input fields }\end{array}$ & 430 & 23.1 \\
\hline $\begin{array}{l}\text { Descriptive text for } \\
\text { tables (captions, } \\
\text { descriptions, table } \\
\text { headings) }\end{array}$ & 292 & 15.7 \\
\hline
\end{tabular}

\section{Invalid Page Code (html/xhtml, css)(Success Criteria H75, H93, H94, G134, G192)}

Incorrect or invalid html/xhtml or css code was contained on $99.8 \%$ of pages checked. Invalid page markup is a significant accessibility concern because screen reading software, along with other assistive technology, works by interpreting the page code. If the page code is incorrect, the software may have problems reading the page properly.

Screen reading software checks the doctype specified in each web page to determine how to interpret the page code. The doctype is a note before the html code that specifies what kind of code the page is written in (e.g., xhtml transitional, $\mathrm{html} 4.0$ strict). If the code specified in the doctype does not match the type of code actually used, the code may not be interpreted properly either by a web browser or by screen reading software. The most common markup problem encountered in this study was a mismatch between the specified doctype and the form of html/xhtml code used to create the page. According to guidelines, web page creators should pick an appropriate doctype and use the conventions required by that type of code.

Doctype mismatch, however, contributed to fewer than half of the markup errors. The other errors consisted of badly formed html code, such as incorrectly used ids or ids that are not unique, bad links, dangling or improperly closed tags, and a variety of other mistakes. The overwhelming prevalence of these types of html mistakes suggested that library web sites do not regularly use a quality control process or include regular page code validation as part of their site development. To achieve accessibility, it is important to have well-formed, valid html markup that is consistent with the doctype specification. To meet WCAG 2.0 guidelines, libraries will need to ensure that page code is valid and to correct errors discovered during validation. Many free online tools exist to help validate page markup, such as the W3C's Markup Validation Service and CSS Validation Service.

\section{Poor Color Contrast (Success Criteria G145, G148, F24, F83)}

A few of the web sites analyzed had consistently good color contrast, but $90.4 \%$ of all pages analyzed had at least one color contrast error. Poor color contrast makes it difficult for people with low vision or color blindness to read what is on the page. WCAG 2.0 requires a minimum contrast ratio of $3: 1$ between foreground and background colors for text sized 18pt or larger, and 4.5:1 for smaller text. The same contrast rules apply for both regular page text and less obvious text, such as visited links, labels, buttons, and text or information in graphics. If font sizes are small, the 
font type is in italics or is otherwise hard to read, or the background is patterned, WCAG 2.0 guidelines require a stronger contrast between background and text to make the text readable. Free tools such as WCAG Contrast Checker, which was used in this study, are available to help test for WCAG 2.0 contrast ratios.

\section{Incorrect Form Control Labelling (Success Criteria H44, H65, H71, H90, G131, G162, G184)}

Problems with incorrect form control labelling were found on $67.9 \%$ of pages.

Although few pages contained a standard web form, nearly all pages did contain a search box, which is considered to be a form field and therefore requires proper form labelling.

Most form label errors encountered in this study were related to two major issues. First, many form fields, especially search boxes, did not have a label. WCAG 2.0 guidelines require labels positioned near form fields so it is clear what the function of the fields are to people who encounter them. If this is not possible, the guidelines specify that form fields should be identified by using a title attribute: for example, <INPUT type="text" name="lastname" id="Iname" title="Last Name">. The title attribute is not displayed on screen but can be read by screen reading software and used to identify the purpose and function of the form field.

Second, many form labels were not properly connected with the form input fields to which they belong. This means that people using screen reading software will have difficulty matching the label with a form field. WCAG 2.0 specifies that form labels should be associated with form fields in the page markup by first creating the form field and giving it an id, then creating the label and specifying that it belongs with the form field's id. For example,

$<$ LABEL for="Iname">Last Name</LABEL >

$<$ INPUT type="text" name="lastname" id="Iname">

\section{Links with the Same Text (Success Criteria G53, H30, H33, H77, H78, H79, H80,} F63, F84)

Multiple links with the same text were contained on $60.1 \%$ of pages. Identical link text is problematic because people using screen reading software commonly navigate using links out of context, reading all the links on the page in a consecutive list. If multiple links have the same text, someone using screen reading software to navigate using links out of context can't determine the difference between the links or where each link might lead to. A very common example of multiple links on a page with the same link text, but pointing to different places, is "more..." According to accessibility guidelines, the purpose of each link should be easily determined by unique link text.

WCAG 2.0 also specifies, however, that if links on a page do have the same text, more information should be provided in a title attribute to distinguish the links from each other. The title attribute is a core element and can be used with nearly all html and xhtml tags. Adding a title to a link works the same way as adding one to a form control: for example, <a title="more about finding books"

href="/books.html">More...</a>. The title attribute will not display on screen but will be read by screen reading software to give users better information about the 
purpose and destination of the link. In some browsers, the title also appears as a pop-up if moused over.

\section{No Alt Attributes for Non-Text Content (Success Criteria G73, G74, G82, G92, G94, H36, H37, H45, H67, F20, F30, F38, F39, F65)}

At least some alt attributes for non-text content were missing from $53.8 \%$ of pages analyzed in this study. Alternate or alt text is critical for accessibility since people with visual impairments have no way to interpret non-text information such as graphics or images, including those used as links or buttons. Screen reading software reads the page markup and knows that there is an image, but unless alt text is added it has no way of knowing what the content of the image is. Therefore, WCAG 2.0 guidelines specify adding alt text to any meaningful non-text content using the alt attribute: for example, <img alt="submit button"

src="/images/button.jpg" $>$. The alt text should describe the purpose and function of the image and should be clear and short.

According to the guidelines, purely decorative images such as lines or spacers or any other images without meaningful content should not have alt text added. Instead, the alt attribute should contain empty double quotes: for example, <img alt="" src="/images/red_line.jpg">. The double quotes let screen reading software know that the alt text has been left purposely blank, and that the image has no meaningful content and can be skipped over. Therefore, WCAG 2.0 guidelines specify that all non-text content needs to include an alt attribute containing either meaningful alt text or empty quotes. If no alt attribute is used at all, the software will not be able to interpret the image properly, and users will feel that they are missing important page content.

\section{Use of HTML for Formatting (Success Criteria G115, G140)}

$\mathrm{Html}$ was used instead of css for formatting in $48.1 \%$ of pages in this study. This is a problem for accessibility, since web pages need to be structured properly to be easily interpreted by assistive software and technologies. WCAG 2.0, along with major web standards, recommends separating a page's structure and content from its presentation. This means that html/xhtml should be used for the page's structure and content: for example, to specify paragraphs, links, lists, and headings. Guidelines specify that css should be used to format and style web page content, rather than html tags like bold and italic. Separating page structure from formatting allows people with low vision to easily adjust the presentation of information on the page so that it is more readable for them: for example, by using different or larger fonts or different colors.

\section{Improper Use of Headings (Success Criteria G130, G141, H42, H69, F43)}

Headings were used incorrectly on $51.1 \%$ of the web pages analyzed. As mentioned, pages need to be properly structured using html code to specify the function of various elements of the page so assistive software and devices can interpret them correctly. Appropriate tags need to be present so assistive software can tell whether certain content is a paragraph, a list, or a heading, for example.

Headings are especially important for accessibility since people using screen reading software navigate extensively using headings. According to WCAG 2.0, headings 
should be used to meaningfully group and distinguish content on a page. Headings are part of the structure of the page and should be given the appropriate tags to distinguish them as headings in the html code (e.g., h1, h2). Screen reading software can't interpret page formatting, so will not recognize text formatted to look larger and bold as a heading unless it has the appropriate heading tags.

According to WCAG 2.0, headings should be used to define the relationships of the information on the page. An h1 heading indicates a main, top-level section; h2 denotes a second-level subsection nested under a top-level section, and h3 denotes a sub-section that is nested under a second-level sub-section. This nested hierarchical structure allows people who use screen reading software to understand the relationships and structure of the information on a page. Guidelines specify, therefore, that headings should be used in their proper nested hierarchy without skipping levels or using certain headings solely for their size or formatting. Since different headings denote specific relationships, improper use will be confusing for people using screen reading software.

\section{Absolute Units of Measure (Success Criteria C12, C13, C14, C28, G146, G178, G179, SCR34)}

Absolute units of measure were found in $39.7 \%$ of pages: for example, using pixels to specify the size of elements on a page. Using absolute units of measure creates a problem for people with low vision, who often use software to enlarge the page to make it easier to read. Web page creators often use pixels or other absolute units of measure for more control over the look of a page. However, since the sizes are absolute, they can't be changed, making it impossible for people to enlarge the page properly. WCAG 2.0 guidelines specify that users should be able to resize pages $200 \%$ without losing content or functionality. This can be done either by rescaling text containers as text is resized or by using a flexible liquid layout that allows the page to be easily resized. In addition, guidelines require web page creators to use relative units of measure, such as percentages, instead of absolute units.

\section{No Submit Buttons for Input Fields (Success Criteria G80, G90, H32, H84)}

Submit buttons for form input fields or controls were missing from $23.1 \%$ of pages. WCAG 2.0 specifies that all forms need to have a submit button, so people have a way to explicitly cause something to happen. Otherwise, people may not be able to tell what they need to do to submit the text they have entered. The most common example of this problem was a search box without a submit button. Search boxes are considered to be form fields and therefore require a submit button of some kind. According to the guidelines, this button should be accessible by keyboard controls as well as a mouse since people using screen reading software or with certain physical disabilities navigate using a keyboard rather than a mouse.

\section{Structure and Descriptive Text for Tables (success criteria H39, H43, H51, H63, H73, F33, F34, F46, F49)}

Proper descriptive text and structural markup for tables was missing in $15.7 \%$ of pages evaluated in this study. However, since a relatively small number of total pages contained tables, this means that nearly all pages with tables had accessibility errors associated with those tables. Unless tables are structured properly, people 
using screen reading software have difficulty reading them. Screen reading software reads all tables linearly, across the whole top row, then across the row beneath it. Unless the table is structured with this linear reading order in mind, the information in the table may make little sense to people using screen reading software.

In WCAG 2.0, page layout tables are allowed but not encouraged. According to the guidelines, tables should mainly be used to present data. Descriptive text, including captions, table summaries, and column headings, should be added to data tables so they can be interpreted more easily by people using screen reading software.

Table captions are displayed in the browser for everyone, but also help screen reading software identify the purpose of the table. Captions can be added using the $<$ caption > tag directly after the <table> tag: for example,

$<$ table $>$

$<$ caption>Opening Hours</caption>

Table descriptions are recommended for more complex tables where the caption would not provide enough information to introduce a reader to the purpose of the table. Descriptions are invisible but help screen reading software orient the reader to the contents or structure of the table before the table content is read. Table summaries can be added by using a summary attribute in the table element: for example, <table summary="A table containing opening hours for the main and branch libraries for the first two weeks of the fall term.">.

Finally, WCAG 2.0 specifies that tables should have column and row headings, and these headings should be identified as headings in the table markup. Heading cells are specified in the page code by using <th $>$ tags to identify the cells as either column or row headings, which helps screen reading software make better sense of the table data. In addition, the scope attribute can be added to <th > cells to identify whether the headers apply to a row or column. For example,

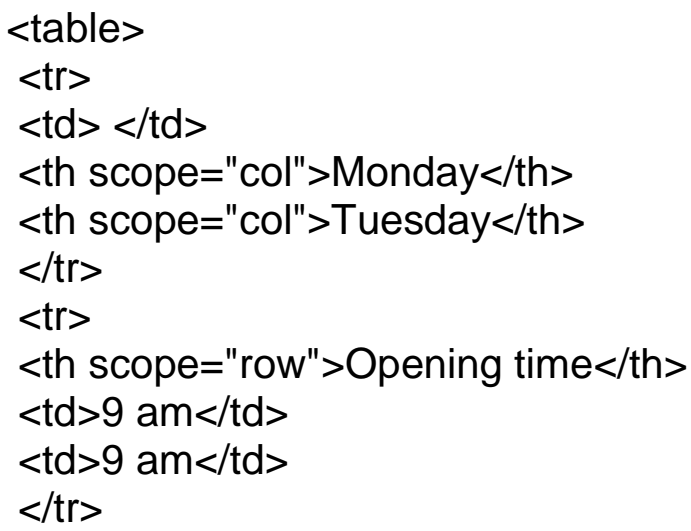

\section{Other Errors: Embedded Content (Success Criteria H46)}

Although a large number of additional accessibility errors were found on web sites assessed in this study, most occurred infrequently or mainly on single sites. One that merits discussion, however, is embedding. Although only $3 \%$ of pages analyzed contained embedded elements, such as video or documents from external sources like Slideshare or Youtube, virtually all of them contained an embedded element error. 
Embedding is one of the rare cases where invalid markup is acceptable in WCAG 2.0. The most commonly used embed methods are not valid markup, but WCAG 2.0 acknowledges that there are no workable accessible alternatives at present. However, according to WCAG 2.0, a <noembed > tag needs to be present within the embed code. This <noembed $>$ alternative should direct people to an accessible alternate format if they are not able to access the embedded content. Using the $<$ noembed $>$ tag therefore requires the creation of an accessible alternative version of embedded material to direct people to. If there is no possibility of creating an accessible alternative version, embedding content without it would not be considered accessible under the WCAG 2.0 regulations.

\section{Other WCAG Guidelines}

WCAG 2.0 contains many guidelines other than those discussed here. The purpose of this research project is to identify the most common errors as identified by automated testing, which have been discussed above. As mentioned, however, many WCAG 2.0 criteria can't be evaluated this way. Only errors that can be determined by analyzing the page code can be identified by automated testing, and many WCAG 2.0 criteria do not involve page code. Some of the more common WCAG 2.0 failures that have appeared in other studies (Stewart, Narendra and Schmetzke; Tatomir and Durrance; Jaeger; Brophy and Craven; Brown-Sica), but which are not easily checked by automated testing of the page code, include:

- Simplicity and usability (success criteria G153, G57, G101, G102): Simple, clear, layout and design is important for accessibility. Busy, cluttered design presents a barrier for people with cognitive or print disabilities (Dermody and Majekodunmi 155).

- Consistency (success criteria G61, G197): WCAG 2.0 guidelines specify consistency in navigation, placement, and wording. Inconsistency creates difficulties for everyone but especially for people with learning or cognitive disabilities.

- Skip navigation (success criteria G1, G123, SCR28): People using screen reading software are forced to go through repetitive content such as navigation and menus on each page. Guidelines recommend adding skip navigation, such as a "hidden" link or other mechanism that allows such users to skip this repetitive content to get quickly to the unique content on each page.

- Keyboard accessibility (success criteria G90, G202, H4, SCR29, SCR35): People with some physical or visual impairments navigate using a keyboard rather than a mouse. WCAG 2.0 specifies that all web content and functions need to be accessible by keyboard. Commonly used navigation keys are tab (for moving from item to item) and enter (to "click").

- Pop-up windows (success criteria G200, G201): People using screen reading software have difficulties navigating from window to window and can be confused by windows that appear without warning. Guidelines recommend not generating new windows unless absolutely necessary, and giving warning in advance if a link will generate a new window.

- Flash/multimedia content (success criteria FLASH1-36): Flash is one of the least accessible formats on the web for people with disabilities (Thatcher 
et al. 91). An entire section of WCAG 2.0 deals with recommendations for making Flash accessible.

- JavaScript (success criteria G90, SCR2, SCR20, SCR35): People using screen reading software often turn off scripts, so WCAG 2.0 specifies that web pages should work if scripts are turned off. In addition, the guidelines recommend that JavaScript code specify keyboard alternates for any mousedependent functions (e.g., mouseover, rollover, hover).

- Page timeouts and time limits (success criteria G5, G75, G105): People who use assistive technologies often need more time than others to navigate and read content on the web (Brown-Sica 18; Carey). Therefore, accessibility guidelines recommend avoiding page timeouts and automatic page refresh.

- Accessible alternate versions (success criteria G136, G190): According to WCAG 2.0, the goal is to have one version of content that is accessible to everyone. If this is not possible, the guidelines allow the option of providing an alternate accessible version of anything non-accessible. This alternate version, however, needs to have the same purpose and content as the original, should be available along with the original, and should be located next to the original so it is easy to find and use. Text is a good accessible alternate format, since it can be easily re-purposed and used with different assistive technologies (Horton 171).

- Document formats: WCAG 2.0 guidelines state that html is the preferred format for documents on the web. Documents in other formats, such as pdf, should be accessible. Pdf documents can range in accessibility from completely inaccessible for those created through scanning to quite accessible for those created from accessible electronic formats.

\section{Conclusion}

This study found that Ontario library web sites, on average, contained 14.75 WCAG 2.0 accessibility errors. Although this number is higher than the findings of other studies, the methods and criteria used to evaluate sites differs so much that the findings are not easily comparable. More accessibility errors were found on library home pages than on other pages. Since home pages are the primary entry point to library resources and services, the higher number of errors found on those pages raises concerns about barriers to access for people with disabilities.

None of the library sites reviewed were compliant with WCAG 2.0 guidelines. Although many different types of WCAG 2.0 compliance errors were identified, most occurred infrequently or only at one or two sites. However, a few accessibility errors were widely found. Foremost among these was invalid or incorrect html and css page code, which makes it difficult for assistive technologies to correctly read the web page. Other common errors included poor contrast between text and background, incorrect form field labelling, failure to provide submit buttons for search boxes, omitting or providing incorrect alt text for images, having multiple links with the same text, using headings improperly, formatting pages with html instead of css, using absolute units of measure to specify the size of elements on a page, and providing descriptive text for tables so people using screen reading software can interpret them properly. 
Although these errors were common to many of the pages evaluated in this study, many other WCAG 2.0 guidelines were not evaluated due to the limitations of the automated testing method used. Automated accessibility checking is a good starting point for identifying potential web accessibility problems. However, since automated tools can only test for some WCAG 2.0 guidelines, additional testing would be required for any site attempting to correct accessibility problems and comply with WCAG 2.0. Studies recommend starting with automated testing but supplementing it with expert and user testing to identify additional errors (Jaeger 178-9; Brophy and Craven 964). Expert testing involves a review of pages by web creators, using methods like a checklist, navigating with a keyboard, resizing pages, using semiautomated tools like the WCAG Contrast Checker, or using screen reading software emulators like Fangs and Web Anywhere. User testing is an important step in identifying the kinds of barriers people with disabilities experience when using a library's web site. Jaeger used multiple methods of testing and found that user testing was the most helpful in identifying accessibility barriers (178).

Web site accessibility is now a legal requirement in Ontario. Although many changes will need to be made to library web sites before they meet legal requirements for accessibility, the work involved will benefit all users. Many of the basic principles of accessibility are also basic principles of good web design. Examples include the concepts of clear, easily navigable, usable web sites, which are fundamental underpinnings of many of the WCAG 2.0 guidelines. Basic web usability and accessibility are inextricably linked. Web usability expert Steve Krug advises that fixing basic usability problems that confuse everyone will also help remove barriers for people with disabilities; trying to fix accessibility problems without addressing basic site usability issues will not have much impact (174-175). Kirkpatrick refers to the importance of usability for accessibility as "two for the price of one" (26). Studies show that usability, especially clear organization and navigation, is the most important factor to focus on when trying to address barriers for people with visual impairments (Brophy and Craven 960). Clear, usable, easy to navigate web sites benefit all users, not just those with disabilities.

In addition, using accessible web standards has benefits for other emerging types of web design. The principles involved in designing accessible web sites are very similar to those used in designing usable mobile web sites. There has been significant movement towards adapting library web sites to be usable on mobile devices such as tablets and smartphones. Designing for accessibility and for mobile devices both involve solving similar problems of how to adapt to users' individual needs for flexibility, the ability to re-purpose content in different ways, interoperability with different technologies, as well as simplicity and usability in interface design (Rowland 18; Harper \& Yesilada xvi). Understanding how to design well for one purpose will help with designing successfully for the other.

Since none of the library web sites examined in this study currently conform to WCAG 2.0, many changes will need to be made before sites can meet the new legal requirements for accessibility. Web accessibility guidelines and standards will need to be incorporated and integrated into the vocabulary, thinking, and processes of web content creators to successfully achieve WCAG 2.0 conformance. Complying with new web accessibility standards will involve a significant change in web development processes. Studies on achieving library web accessibility have been few in number, and there will be a need for future research and publication to assist 
people in making these web accessibility improvements and in maintaining awareness of progress and developments in the area of web accessibility.

\section{Works Cited}

Brophy, Peter, and Jenny Craven. "Web Accessibility." Library Trends 55.4 (2007): 950-72. Print.

Brown-Sica, Margaret. "Conducting Web Usability Testing with Students with Disabilities." Colorado Libraries 33.4 (2007): 16-9. Print.

Byerley, Suzanne L., Mary Beth Chambers, and Mariyam Thohira. "Accessibility of Web-Based Library Databases: The Vendors' Perspectives in 2007." Library Hi Tech 25.4 (2007): 509-27. Print.

Carey, Kevin. "Accessibility: The Current Situation and New Directions." Ariadne 44 (2005). Web. <http://www.ariadne.ac.uk/issue44/carey/>.

Comeaux, David, and Axel Schmetzke. "Web Accessibility Trends in University Libraries and Library Schools." Library Hi Tech 25.4 (2007): 457-77. Print.

Dermody, Kelly, and Norda Majekodunmi. "Online Databases and the Research Experience for University Students with Print Disabilities." Library Hi Tech 29.1 (2011): 149-160. Print.

Hackett, S., B. Parmanto, and Z. Xiaoming. "A Retrospective Look at Website Accessibility Over Time." Behaviour \& Information Technology 24.6 (2005): 40717. Print.

Hein, Karen, and John Seyfarth. "Developing Accessible Web Pages: WAI and Section 508 Compliance Tips." Nebraska Library Association Quarterly 38.2 (2007): 22-6. Print.

Horton, Sarah. Access by Design: A Guide to Universal Usability for Web Designers. Berkeley, CA: New Riders, 2006. Print.

Jaeger, Paul T. "Assessing Section 508 Compliance on Federal e-Government Web Sites: A Multi-Method, User-Centered Evaluation of Accessibility for Persons with Disabilities." Government Information Quarterly 23.2 (2006): 169-90. Print.

Kirkpatrick, Cheryl H. "Getting Two for the Price of One: Accessibility and Usability." Computers in Libraries 23.1 (2003): 26-9. Print.

Krug, Steve. Don't Make Me Think: A Common Sense Approach to Web Usability. $2^{\text {nd }}$ ed. Berkeley, CA: New Riders, 2006. Print.

Murley, Diane. "Web Site Accessibility." Law Library Journal 100.2 (2008): 401-6. Print. 
Ontario Ministry of Community and Social Services. "Integrated Accessibility Standards: Ontario Regulation 191/11, made under the Accessibility for Ontarians with Disabilities Act, 2005." 2011. Web. <http://www.e-

laws.gov.on.ca/html/source/regs/english/2011/elaws src regs r11191 e.htm>.

---. "Understanding Accessibility." 2011.Web.

$<$ http://www.mcss.gov.on.ca/en/mcss/programs/accessibility/understanding acces sibility/>.

Power, Rebecca, and Chris LeBeau. "How Well Do Academic Library Web Sites Address the Needs of Database Users with Visual Disabilities?" The Reference Librarian 50.1 (2009): 55-72. Print.

Providenti, Michael and Robert Zai. "Web Accessibility at Kentucky's Academic Libraries." Library Hi Tech 24.4 (2007): 478-493. Print.

Rowland, C., et al. "Universal Design for the Digital Environment: Transforming the Institution." EDUCAUSE Review 45.6 (2010). Web.

$<$ http://www.educause.edu/EDUCAUSE+Review/EDUCAUSEReviewMagazineVol ume45/UniversalDesignfortheDigitalEn/218700>.

Schmetzke, Axel. "Introduction: Accessibility of Electronic Information Resources for All." Library Hi Tech 25.4 (2007): 454-6. Print.

Shaughnessy, Peggy. "Advocating for Accessibility in the Next Generation Catalog." Colorado Libraries 33.4 (2007): 29-34. Print.

Spindler, Tim. "The Accessibility of Web Pages for Mid-Sized College and University Libraries." Reference \& User Services Quarterly 42.2 (2002): 149-54. Print.

Stewart, Ron, Vivek Narendra, and Axel Schmetzke. "Accessibility and Usability of Online Library Databases." Library Hi Tech 23.2 (2005): 265-86. Print.

Tatomir, Jennifer, and Joan C. Durrance. "Overcoming the Information Gap: Measuring the Accessibility of Library Databases to Adaptive Technology Users." Library Hi Tech 28.4 (2010): 577-94. Print.

Thatcher, Jim, et al. Web Accessibility: Web Standards and Regulatory Compliance. Berkeley, CA: Apress, 2006. Print.

World Wide Web Consortium (W3C). "Techniques and Failures for Web Content Accessibility Guidelines 2.0." 2010. Web. <http://www.w3.org/TR/WCAG20$\underline{\text { TECHS/>. }}$

---. "Web Content Accessibility Guidelines (WCAG) 2.0." 2008. Web. <http://www.w3.org/TR/WCAG20/>. 